\title{
Pancreatite intersticial crônica em bovino causada por Eurytrema coelomaticum
}

\author{
Chronic interstitial pancreatitis in cattle caused by Eurytrema coelomaticum
}

\author{
Pedro de Souza Quevedo ${ }^{I^{*}}$ Mariana Mendes $^{\mathrm{I}}$ Felipe Geraldo Pappen ${ }^{\mathrm{II}}$ \\ Mauro Pereira Soares ${ }^{\mathrm{III}}$ Gertrud Muller ${ }^{\mathrm{IV}}$ Nara Amélia da Rosa Farias ${ }^{\mathrm{IV}}$
} \begin{abstract}
Descrevem-se os achados clínicos e patológicos
atribuídos à infecção maciça por trematódeos pancreáticos do RESUMO gênero Eurytrema (Loos, 1907) em uma novilha da raça Jersey, procedente do município de Seara, região oeste do estado de Santa Catarina, Brasil. $O$ animal de dois anos de idade apresentava desenvolvimento insuficiente, emagrecimento crônico, caquexia, decúbito prolongado e anemia, sendo submetido à eutanásia in extremis. Na necropsia observou-se aumento de volume do pâncreas, que estava esbranquiçado, crepitante, com a superfície de corte irregular, onde foram visualizados inúmeros trematódeos emergindo ativamente do órgão. Histologicamente havia pancreatite intersticial crônica. O parasito foi identificado como Eurytrema coelomaticum (Giard \& Billet, 1882). Trata-se do primeiro registro de euritrematose clínica em bovino no estado de Santa Catarina.
\end{abstract}

Palavras-chave: Euritrematose clínica, histopatologia, trematoda.

\section{ABSTRACT}

This study describes the clinical and pathological findings attributed to massive infection by pancreatic trematodes of the genre Eurytrema (Loos, 1907) in a female Jersey heifer from the city of Seara located in the west part of Santa Catarina state, Brazil. The two-year-old animal showed insufficient development, chronic emaciation, cachexia, prolonged decubitus and anemia being euthanized in extremis. The necropsy indicated an enlarging of the pancreas which demonstrated crepitated pale surface of irregular cut and, where several trematodes were observed emerging actively from the organ. Histologically, there was chronic interstitial pancreatitis. The parasite was identified as Eurytrema coelomaticum (Giard \& Billet, 1882). It is the first record of clinical eurytrematosis in bovines in the state of Santa Catarina.

Key words: Clinical eurytrematosis, histopathology, trematoda.
Eurytrema pancreaticum (Janson, 1889) e E. coelomaticum (Giard \& Billet, 1882) são parasitos encontrados nos ductos pancreáticos e ocasionalmente em ductos biliares e intestino delgado dos ruminantes (JUBB 1993, ILHA et al., 2005, BASSANI et al., 2006, BASSANI et al., 2007). $\boldsymbol{E}$. pancreaticum é comumente relatado em ovinos na ilha de Sumatra, na Indonésia (DORNY et al., 1996). E. coelomaticum é um trematódeo considerado achado incidental, usualmente observado durante o abate e em necropsias, em diversos estados brasileiros (ILHA et al., 2005, BASSANI et al., 2006, RACHID et al., 2011). A euritrematose bovina está associada à queda de desempenho produtivo dos animais, gerando também perdas à indústria de extração de insulina, pois implica a condenação do pâncreas durante inspeções de rotina (BASSANI et al., 2007, RACHID et al., 2011).

As espécies do gênero Eurytrema possuem ciclo heteroxeno, o hospedeiro definitivo é infectado pela ingestão acidental de gafanhotos contendo metacercárias, as quais são liberadas dos cistos no duodeno, migrando posteriormente ao pâncreas (MATTOS Jr \& VIANNA 1987, JUBB 1993).

Relata-se neste trabalho um caso clínico de euritrematose, caracterizado por emaciação crônica atribuída à infecção maciça por trematódeos pancreáticos do gênero Eurytrema. O diagnóstico

\footnotetext{
IPrograma de Pós-graduação em Parasitologia, Universidade Federal de Pelotas (UFPel), 96001-970, Pelotas, RS, Brasil. E-mail: pedrosquevedo@hotmail.com.*Autor para correspondência.

${ }^{\text {IIC }}$ urso de Veterinária, Instituto Federal Catarinense (IFC), campus Concórdia, Concórdia, SC, Brasil.

IIILaboratório Regional de Diagnóstico (LRD), UFPel, Pelotas, RS, Brasil.

${ }^{\text {IV }}$ Departamento de Parasitologia, UFPel, Pelotas, RS, Brasil.
} 
foi realizado em uma novilha da raça Jersey, no município de Seara, região oeste do estado de Santa Catarina.

Ao exame clínico o animal apresentava letargia, fraqueza, prostração, depressão, caquexia, anemia e decúbito prolongado, apesar do manejo nutricional adequado. Os sinais clínicos eram semelhantes aos relatados por outros autores para bovinos com euritrematose no Brasil (TRAVASSOS 1944, ILHA et al., 2005, BASSANI et al., 2006, RACHID et al., 2011). O animal apresentava condições corporais insatisfatórias, ainda que desfrutasse de áreas com forragem de boa qualidade. Neste estudo, segundo relato do proprietário, o animal passou a ter retardo no crescimento e emagrecimento progressivo, que perdurou por cerca de um ano. $\mathrm{O}$ decréscimo no desenvolvimento persistiu mesmo após sucessivos tratamentos com anti-helmínticos e suplementação nutricional.

Diante do agravamento do quadro, o animal foi submetido à eutanásia para realização da necropsia. Foram coletados fragmentos de órgãos das cavidades torácica, abdominal e sistema nervoso central, os quais foram fixados em formol tamponado a $10 \%$, encaminhados ao laboratório, processados rotineiramente e corados por hematoxilina-eosina (HE). Os parasitos coletados do pâncreas no momento da necropsia foram também fixados em formol tamponado a $10 \%$. O material foi encaminhado ao Laboratório de Parasitologia da Universidade Federal de Pelotas (UFPel), onde foi preparado para a identificação, de acordo com a técnica preconizada por AMATO \& AMATO (2009).

Durante a necropsia foi observado aumento do pâncreas que estava esbranquiçado, com superfície irregular (Figura $1 \mathrm{~A}$ ) e crepitante. Ao corte do órgão foram visualizados inúmeros trematódeos emergindo ativamente. Os ductos pancreáticos estavam espessados, esbranquiçados, dilatados e com paredes fibrosas (Figura $1 \mathrm{~B}$ ). Esse aspecto do pâncreas é semelhante ao descrito em relatos clínicos de euritrematose realizados no Paraná e em Minas Gerais (ILHA et al., 2005, RACHID et al., 2011).

Os achados histológicos permitiram a diferenciação de três padrões morfológicos de lesão pancreática. O primeiro, correspondente à lesão inicial, era caracterizado por infiltrado inflamatório com predominância de macrófagos, linfócitos e eosinófilos circundando ductos pancreáticos hiperplásicos contendo ovos em sua luz (Figura 2A). O segundo estágio evidenciou a formação de múltiplos focos inflamatórios em torno de coleções de ovos, delimitados por discreta camada de fibroblastos. Esses pequenos sítios formavam granulomas coalescentes (Figura $2 \mathrm{~B}$ ). O terceiro e mais avançado estágio consistia na confluência das lesões observadas no estágio anterior com destruição dos ductos pancreáticos. Coleções maiores de ovos e infiltrado inflamatório linfohistiocitário eram evidentes (Figura $2 \mathrm{C}$ ). Essas áreas eram circundadas por espessa camada de tecido conjuntivo fibroso. O tecido remanescente era composto por ductos dilatados e hiperplásicos, repletos de parasitos adultos (Figura 3). Essas lesões são semelhantes às observadas por ILHA et al., (2005).

As secções histológicas e as preparações permanentes, coradas com Carmim, permitiram a identificação de estruturas típicas de Eurytrema coelomaticum (Figura 4). Foram realizadas medições dos parasitas e os valores médios obtidos foram: $3,85 \mathrm{~mm}$ de comprimento, 2,42mm de largura do corpo, $0,5 \mathrm{~mm}$ de diâmetro da ventosa oral e

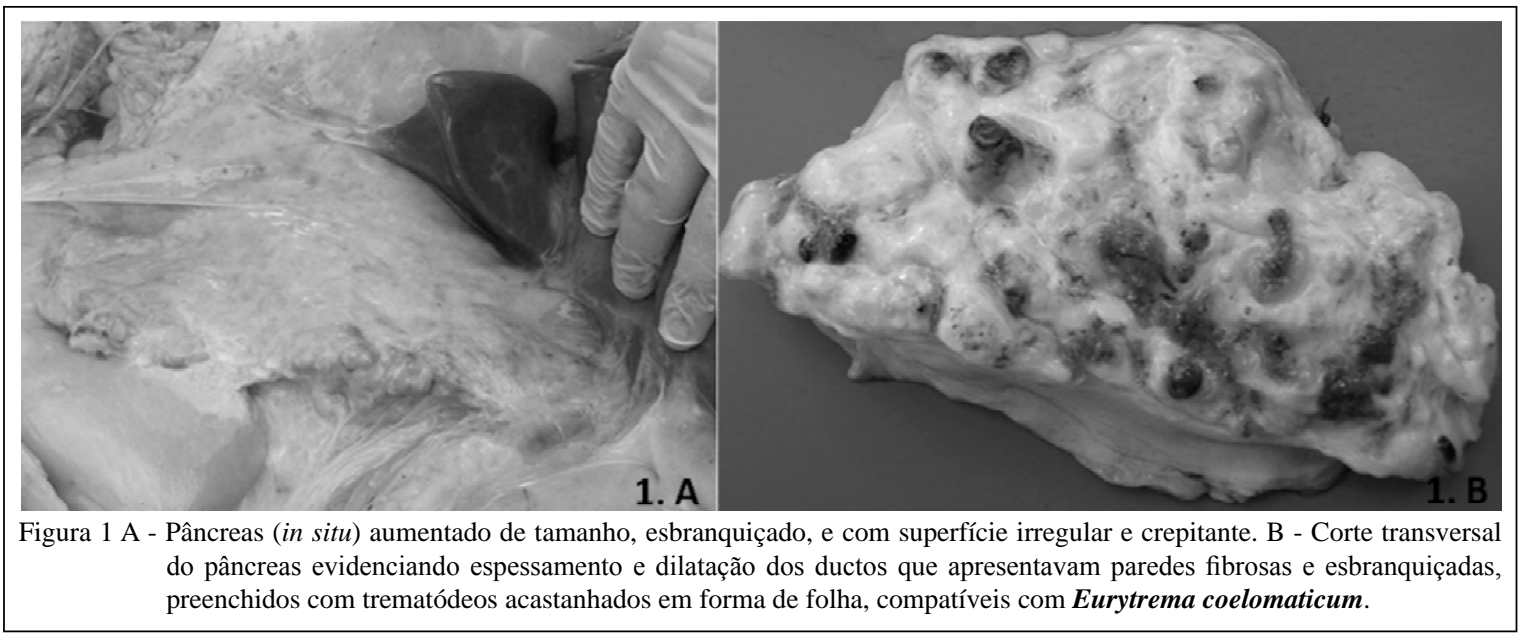

Ciência Rural, v.43, n.8, ago, 2013. 


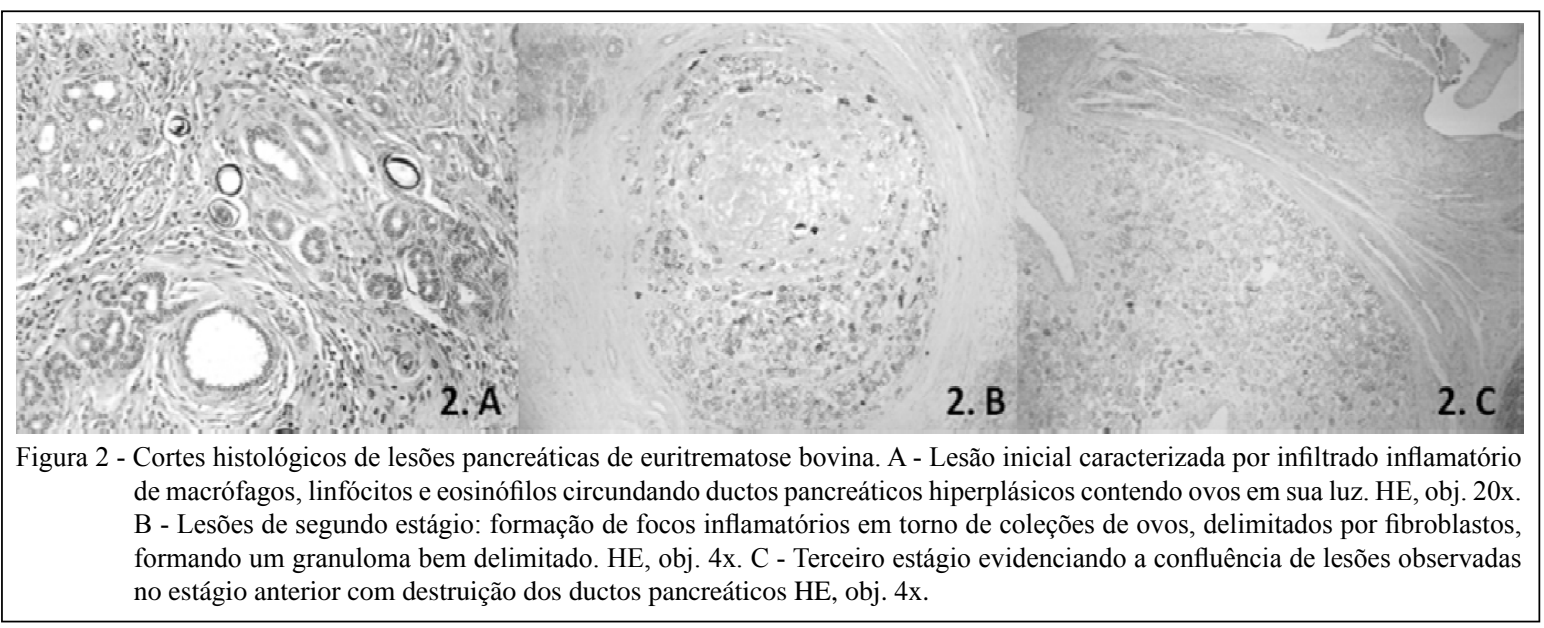

$0,58 \mathrm{~mm}$ do acetábulo, $0,2 \mathrm{~mm}$ de comprimento da faringe e $0,04 \mathrm{~mm}$ de comprimento e $0,02 \mathrm{~mm}$ de largura dos ovos. Esses valores são inferiores aos descritos para $\boldsymbol{E}$. coelomaticum sendo essa diferença atribuída, em parte, ao fato de os espécimes terem sido acondicionados em formol, o que promoveu a contração das estruturas dos trematódeos (TRAVASSOS 1944, ILHA et al., 2005).

As técnicas de contagem de ovos de Eurytrema eliminados pelas fezes de hospedeiros definitivos parasitados são trabalhosas e a probabilidade de falsos negativos é considerável (BELÉM et al., 1993).

Essa dificuldade no diagnóstico é particularmente importante para animais pouco parasitados que eliminam ovos no ambiente constantemente. Devido a essa limitação no diagnóstico parasitológico, a necropsia é uma ferramenta importante em casos de euritrematose (ILHA et al., 2005).

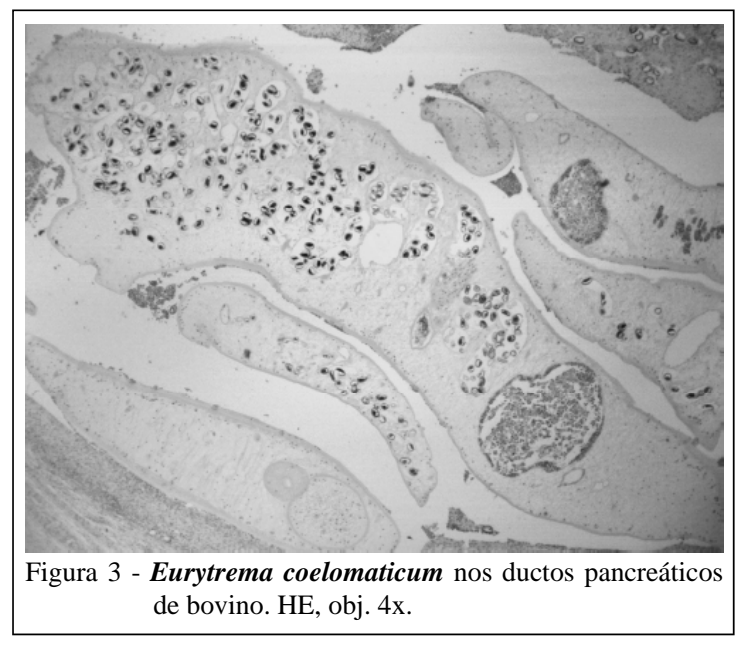

O diagnóstico de insuficiência pancreática, associada com fibrose induzida por E. coelomaticum, deve ser considerado na diferenciação das doenças que acometem bovinos e podem determinar perda de peso crônica. Entre esses, podem ser destacadas as intoxicações por plantas contendo alcaloide pirrolizidínico (LUCENA et al., 2010).

São desconhecidas drogas anti-helmínticas capazes de combater o parasitismo por Eurytrema (ILHA et al., 2005, BASSANI et al., 2006). Logo, os danos causados por esse parasito são irreversíveis. Embora casos clínicos sejam raramente observados nos rebanhos, a alta letalidade em animais clinicamente afetados preocupa pecuaristas (AZEVEDO et al., 2005, BASSANI et al., 2007). Diante dessa realidade, conhecer os locais onde o parasito ocorre e as peculiaridades de seu ciclo são informações fundamentais.

Trata-se do primeiro registro de euritrematose clínica em bovino no estado de Santa Catarina. Cumpre salientar que o animal era nascido e criado na região sudoeste do estado. Novos relatos e estudos aprimorando o conhecimento acerca da ocorrência da dinâmica de hospedeiros intermediários

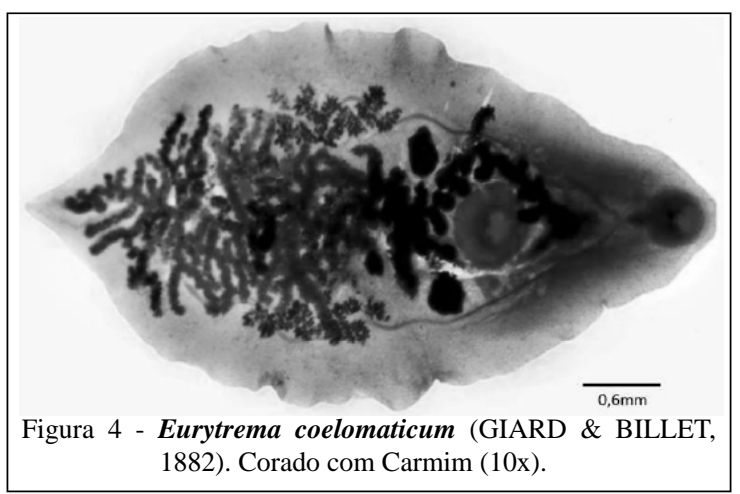


de $\boldsymbol{E}$. coelomaticum são pertinentes para que sejam adotadas medidas de controle, que são a única forma conhecida de minimizar impactos causados por este trematódeo. Tais medidas consistem em reduzir ambientes propícios à proliferação de hospedeiros intermediários e/ou impedir que bovinos pastem em áreas onde estes ocorrem.

\section{COMITÊ DE ÉTICA E BIOSSEGURANÇA}

O procedimento de eutanásia foi realizado com a devida permissão do proprietário e de acordo com as normas preconizadas pelo Colégio Brasileiro de Experimentação Animal (COBEA).

\section{REFERÊNCIAS}

AMATO, J. F. R. \& AMATO, S. B. Técnicas gerais para coleta e preparação de helmintos endoparasitos de aves. In: Von MATTER, S. et al. 2009. Ornitologia e conservação: ciência aplicada, técnicas de pesquisa e levantamento. Rio de Janeiro, RJ. Technical Books, 516p. 2009

AZEVEDO, J.R., et al. Prevalência e distribuição geográfica de euritrematose bovina em animais abatidos no norte do Estado do Paraná. Pesquisa Veterinária Brasileira. 24, 2326, 2005. Disponível em: <http://dx.doi.org/10.1590/S0100736X2004000100006>. Acesso em: 28 de Julho de 2012.

BASSANI, C.A. et al. Epidemiology of eurytrematosis (Eurytrema spp. Trematoda: Dicrocoeliidae) in slaughtered beef cattle from the central-west region of the State of Paraná, Brazil. Veterinary Parasitology. 141, 356-361, 2006. Disponível em: <http://www. sciencedirect.com/science/article/pii/S0304401706003487>. Acesso em: 24 de Julho de 2012. http://dx.doi.org/10.1016/j. vetpar.2006.06.003

BASSANI, C.A. et al. Euritrematose bovina. Semina: Ciências Agrárias, v. 28, 299-316, 2007. Disponível em: <http://www. uel.br/revistas/uel/index.php/semagrarias/article/view/3443/0>. Acesso em: 29 de Julho de 2012.

BELÉM P.A.D. et al. Distribution of the number of Eurytrema sp. eggs per gram of feces in naturally infected cattle. Veterinary
Parasitology. 49 325-330, 1993. Disponível em: <http://www. sciencedirect.com/science/article/pii/0304401793901316>. Acesso em: 23 de Julho de 2012. doi.org/10.1016/03044017(93)90131-6

DORNY P. et al. 1996. Helminth infections of sheep in North Sumatra, Indonesia. Veterinary Parasitology, 61 353-358, 1996. Disponível em: <http://www.sciencedirect.com/science/article/ pii/0304401795008268>. Acesso em: 03 de Agosto de 2012. doi. org/10.1016/0304-4017(95)00826-8

ILHA, M.R.S. et al. Wasting and mortality in beef cattle parasitized by Eurytrema coelomaticum in the State of Paraná, southern Brazil. Veterinary Parasitology, 133, 49-60, 2005. Disponível em: <http://www.sciencedirect.com/science/article/pii/ S0304401705001779>. Acesso em: 30 de Julho de 2012. http:// dx.doi.org/10.1016/j.vetpar.2005.02.013

JUBB, K.V.F. The pancreas. In: Jubb, K.V.F., Kennedy, P.C., Palmer, N. (Eds.). Pathology of Domestic Animals. 4a ed., vol. 2. Academic Press, pp. 407-424, 1993. 3rd ed., pp. 183-266. Academic Press, San Diego, CA

LUCENA, R. B. et al. Intoxicação por alcaloides pirrolizidínicos em ruminantes e equinos no Brasil. Pesquisa Veterinária Brasileira. 30(5):447-452, maio 2010. Disponível em: 〈http://www.scielo.br/ scielo.php?pid=S0100-736X2010000500013\&script=sci_arttext $>$. Acesso em 02 de Agosto de 2012. http://dx.doi.org/10.1590/ S0100-736X2010000500013.

MATTOS JR, D.G. \& VIANNA, S.S.S. O Eurytrema coelomaticum (Trematoda: Dicrocoeliidae) no Brasil. Arquivos Fluminenses de Medicina Veterinaria, v.2, n.1, p.3-7, 1987. Disponível em: <http://agris.fao.org/agris-search/search/display. doi.f=2012/OV/OV201209921009921.xml;BR19890007479>. Acesso em: 01 de Agosto de 2012.

RACHID, M.A. et al. Pancreatite crônica intersticial e caquexia causada por Eurytrema coelomaticum em vaca Nelore. Arq. Bras. Med. Vet. Zootec., v.63, n.3, p.741-743, 2011. Disponível em: <http://www.scielo.br/scielo.php?script=sci_arttext\&pid =S0102-09352011000300028>. Acesso em: 23 de Julho de 2012. http://dx.doi.org/10.1590/S0102-09352011000300028

TRAVASSOS, L. Revisão da família Dicrocoeliidae, Odhner, 1910. 1944. 357 p. illus. Monographias do Instituto Oswaldo Cruz Instituto Oswaldo Cruz, RJ. 